\title{
Can There be Given Any Meaning to Contextuality Without Incompatibility?
}

\section{Andrei Khrennikov ${ }^{1}$}

Received: 19 May 2020 / Accepted: 16 November 2020 / Published online: 2 December 2020

(C) The Author(s) 2020

\begin{abstract}
Our aim is to compare the fundamental notions of quantum physics - contextuality vs. incompatibility. One has to distinguish two different notions of contextuality, Bohrcontextuality and Bell-contextuality. The latter is defined operationally via violation of noncontextuality (Bell type) inequalities. This sort of contextuality will be compared with incompatibility. It is easy to show that, for quantum observables, there is no contextuality without incompatibility. The natural question arises: What is contextuality without incompatibility? (What is "dry-residue"?) Generally this is the very complex question. We concentrated on contextuality for four quantum observables. We shown that, for "natural quantum observables", contextuality is reduced to incompatibility. But, generally contextuality without incompatibility may have some physical content. We found a mathematical constraint extracting the contextuality component from incompatibility. However, the physical meaning of this constraint is not clear. In Appendix 1, we briefly discuss another sort of contextuality based on Bohr's contextuality-incompatibility principle. Bohr-contextuality plays the crucial role in quantum foundations. Incompatibility is, in fact, a consequence of Bohr-contextuality. Finally, we remark that outside of physics, e.g., in cognitive psychology and decision making Bell-contextuality distilled of incompatibility can play the important role.
\end{abstract}

Keywords Contextuality · Incompatibility · Complementarity principle Joint probability distribution $\cdot$ Noncontextual inequalities $\cdot$ Product of commutators

\section{Introduction}

Contextuality formalized in the form of violation of noncontextuality inequalities, Bellcontextuality $[1,2]$, is a hot topic in quantum physics (see, e.g., [3, 4] and references herein). Unfortunately, it is typically presented in the mathematical framework and its physical meaning is unclear.

Andrei Khrennikov

andrei.khrennikov@1nu.se

1 International Center for Mathematical Modeling in Physics and Cognitive Sciences,

Linnaeus University, Växjö, SE-351 95, Sweden 
We stress that, in fact, one has to distinguish two different notions of contextuality, Bohrcontextuality and Bell-contextuality. In this paper, we consider the latter, see Appendix 2 for for the former. Therefore we shall speak simply about contextuality (having in mind Bell-contextuality).

We point out that discussions on this sort contextuality are typically started with heuristically more attractive definition going back to Bell $[1,2]$. It can be called explicit contextuality: if $A, B, C$ are three quantum observables, such that Acompatible with $B$ and $C$, a measurement of $A$ might give different result depending upon whether $A$ is measured with $B$ or with $C$. However, for incompatible observables $B$ and $C$, this statement is not testable experimentally. ${ }^{1}$ Therefore we proceed with Bellcontextuality that is straightforwardly coupled to experiment. (This approach to contextuality was actively driven by Adan Cabello; so it may be natural to call it Bell-Cabello contextuality.)

It is easy to show that, for quantum observables, there is no contextuality without incompatibility: for compatible observables, it is impossible to violate any noncontextuality inequality (Theorem 1, Section 2). The natural question arises:

Has contextuality without incompatibility any physical meaning?

Generally this is the very complex question. I do not know the answer to it for general noncontextuality inequalities. And I hope that this paper would stimulate foundational research in this direction. We concentrate on contextuality for four quantum observables noncontextuality analog of the CHSH-inequality.

We proved that, for "natural quantum observables" , contextuality is reduced to incompatibility $^{2}$ (in [9-11], the same conclusion was obtained for quantum nonlocality, cf. [12-16]).

At the same time, we shown that generally contextuality without incompatibility may have some physical content. We found a mathematical constraint extracting the contextuality component from incompatibility. However, the physical meaning of this constraint is not clear.

We also remark that there exist positive answers to the inverse question: there can be (non-quantum, quasi-classical) incompatibility without contextuality; as exposed by finite automata [21] as well as for generalized urn models [22], see [23].

In appendix, we briefly discuss another sort of contextuality that is understood more generally in accordance with the Bohr message [24] that all experimental arrangement (experimental context) has to be taken into account in the process of measurement; he pointed to "the impossibility of any sharp separation between the behavior of atomic objects and the interaction with the measuring instruments which serve to define the conditions under which the phenomena appear." Here "phenomenon" is understood as the individual output of measurement [10, 24-26]. Bohr-contextuality is the basis of quantum foundations. Moreover, this is the root of the Bohr's complementarity principle. Incompatibility is a consequence of contextuality, but the latter has to be understood as Bohr-contextuality, Appendix 1 (see also [27]).

\footnotetext{
${ }^{1}$ Svozil proposed to appeal to counterfactuals to design an experimental test for explicit contextuality, see [5-8] for such a test. Unfortunately, he did not elaborate this framework and these papers are practically forgotten.

${ }^{2}$ Outside of physics, e.g., in cognitive psychology and decision making contextuality distilled of incompatibility can play the important role [17-20].
} 


\section{Quantum Theory: Bell-contextuality vs. Bohr-incompatibility}

In this paper, we consider dichotomous observables taking values \pm 1 .

We follow paper [4] (one of the best and clearest representations of contextuality). Consider a set of observables $\left\{X_{1}, \ldots, X_{n}\right\}$; a context $C$ is a set of indexes such that $X_{i}, X_{j}$ are compatible for all pairs $i, j \in C$. A contextuality structure for these observables is given by a set of contexts $\mathcal{C}=\{C\}$, or simply the maximal contexts. For each context $C$, we measure pairwise correlations for observables $X_{i}$ and $X_{j}$ with indexes $i, j \in C$ as well as averages $\left\langle X_{i}\right\rangle$ of observables $X_{i}$. The $n$-cycle contextuality scenario is given by $n$ observables $X_{1}, \ldots, X_{n}$ and the set of maximal contexts

$$
\mathcal{C}_{n}=\left\{\left\{X_{1}, X_{2}\right\}, \ldots,\left\{X_{n-1}, X_{n}\right\},\left\{X_{n}, X_{1}\right\}\right\} .
$$

Statistical data associated with this set of contexts is given by the collection of averages and correlations:

$$
\left\{\left\langle X_{1}\right\rangle, \ldots .\left\langle X_{n}\right\rangle ;\left\langle X_{1} X_{2}\right\rangle, \ldots,\left\langle X_{n-1} X_{n}\right\rangle,\left\langle X_{n}, X_{1}\right\rangle\right\} .
$$

Theorem 1 from paper [4] describes all tight noncontextuality inequalities. In particular, for $n=4$ we have inequality:

$$
\left|\left\langle X_{1} X_{2}\right\rangle+\left\langle X_{2} X_{3}\right\rangle+\left\langle X_{3} X_{4}\right\rangle-\left\langle X_{4} X_{1}\right\rangle\right| \leq 2 .
$$

Theorem 2 [4] demonstrates that, for $n \geq 4$ (cf. Appendix 1 for $n=3$ ), aforementioned tight noncontexuality inequalities are violated by quantum correlations. But,

what is the physical root of quantum violations?

Unfortunately, the formal mathematical calculations [4] used to show violation of noncontextuality inequalities for quantum observables do not clarify physics behind these violations.

Let us turn to the quantum physics, i.e., $X_{1}, \ldots, X_{n}$ are not arbitrary observables, but quantum physical ones. In the quantum formalism, they are represented by Hermitian operators $\hat{X}_{1}, \ldots, \hat{X}_{n}$. Denote the orthogonal projectors onto the corresponding eigenspaces by the symbols $\hat{E}_{j \alpha}, \alpha= \pm 1$.

Suppose now that these observables are compatible with each other, i.e., any two observables $X_{i}, X_{j}$ can be jointly measurable, so in the operator formalism, $\left[\hat{X}_{i}, \hat{X}_{j}\right]=0$. The quantum theory has one amazing feature that is not so widely emphasized:

Pairwise joint measurability implies $k$-wise joint measurability for any $k \leq n$.

If all pairs can be jointly measured, then even any family of observables $\left\{X_{i_{1}}, \ldots, X_{i_{k}}\right\}$ can be jointly measured as well. In principle, there is no reason for this. This is the specialty of quantum theory.

The joint probability distribution (JPD) of compatible observables is defined by the following formula [28]:

$$
P_{i_{1} \ldots i_{k}}\left(\alpha_{i_{1}}, \ldots, \alpha_{i_{k}}\right)=\operatorname{Tr} \rho \hat{E}_{i_{1} \alpha_{i_{1}}} \cdots \hat{E}_{i_{k} \alpha_{i_{k}}} .
$$

In particular, by setting $k=n$ we obtain JPD of all observables,

$$
P_{1 \ldots n}\left(\alpha_{1}, \ldots, \alpha_{n}\right)=\operatorname{Tr} \rho \hat{E}_{1 \alpha_{1}} \cdots \hat{E}_{n \alpha_{n}} .
$$

We remark that the probability distributions given by (4) can be obtained from the latter JPD as the marginal probability distributions:

$$
P_{i_{1} \ldots i_{k}}\left(\alpha_{i_{1}}, \ldots, \alpha_{i_{k}}\right)=\sum_{\alpha_{j}, j \neq i_{1} \ldots i_{k}} P_{1 \ldots n}\left(\alpha_{1}, \ldots, \alpha_{n}\right) .
$$


This formula implies as well that the marginals of JPD $P_{i_{1} \ldots i_{k}}$ of the rank $k$ generate JPDs of the rank $k-1$. In particular, we have the consistency rules for JPDs of ranks 2 and 1 ,

$$
P_{i}\left(\alpha_{i}\right)=\sum_{\alpha_{j}} P_{i j}\left(\alpha_{i}, \alpha_{j}\right)
$$

(in quantum physics, this condition is known as no signaling), and ranks 3 and 2 consistency:

$$
P_{i j}\left(\alpha_{i}, \alpha_{j}\right)=\sum_{\alpha_{k}} P_{i j k}\left(\alpha_{i}, \alpha_{j}, \alpha_{k}\right)
$$

We have the classical probability framework; the Kolmogorov probability model with the probability measure $P \equiv P_{1 \ldots n}$. In this classical probabilistic framework we can prove any noncontextuality inequality (any Bell-type inequality, cf. [29-36], [26, 27]). It is impossible to violate them for compatible quantum observables. We can formulate this result as a simple mathematical statement:

Theorem 1 For quantum observables $X_{1}, \ldots, X_{n}$, (Bell-)contextuality implies incompatibility of at least two of them.

Thus, there is no Bell-contextuality without incompatibility. Does the latter contain something more than incompatibility?

Finally, we remark that noncontextuality inequalities started to be used in applications outside of physics, e.g., in psychology, cognitive science, and decision making [17-20]. If one does not assume that observables are represented by Hermitian operators in Hilbert space, then "no-go" Theorem 1 loses its value.

\section{Is Contextuality Reduced to Incompatibility?}

In [9], I analyzed in details the CHSH-inequality; the CHSH-correlation has the form:

$$
\Gamma=\left\langle A_{1} B_{1}\right\rangle+\left\langle A_{1} B_{2}\right\rangle+\left\langle A_{2} B_{1}\right\rangle-\left\langle A_{2} B_{2}\right\rangle,
$$

where observables $A_{i}$ are compatible with observables $B_{j}, i, j=1,2$. In [9], the tensor product structure of the state space was not explored and quantum observables were represented by Hermitian operators $\hat{A}_{i}, \hat{B}_{j}$ acting an arbitrary Hilbert space. In this framework the CHSH-inequality can be treated as the noncontextuality inequality for four observables; by setting in (9) $A_{2}=X_{1}, B_{1}=X_{2}, A_{1}=X_{3}, B_{2}=X_{4}$, we obtain the correlation:

$$
\Gamma=\left\langle X_{1} X_{2}\right\rangle+\left\langle X_{2} X_{3}\right\rangle+\left\langle X_{3} X_{4}\right\rangle-\left\langle X_{4} X_{1}\right\rangle \text {, }
$$

since we work with quantum observables, we proceed under the compatibility assumption

$$
\left[\hat{X}_{1}, \hat{X}_{2}\right]=0,\left[\hat{X}_{3}, \hat{X}_{2}\right]=0,\left[\hat{X}_{3}, \hat{X}_{4}\right]=0,\left[\hat{X}_{1}, \hat{X}_{4}\right]=0 \text {. }
$$

Now set

$$
\hat{M}_{13}=i\left[\hat{X}_{1}, \hat{X}_{3}\right] \text { and } \hat{M}_{34}=i\left[\hat{X}_{2}, \hat{X}_{4}\right] .
$$

These are Hermitian operators, so they represent some quantum observables $M_{13}$ and $M_{34}$. We remark that these observables are compatible:

$$
\left[\hat{M}_{13}, \hat{M}_{34}\right]=0 .
$$

The following theorem is the noncontextuality reinterpretation of the main result of paper [9]: 


\section{Theorem 2 Condition}

$$
\hat{M}_{13} \circ \hat{M}_{34} \neq 0 .
$$

is necessary and sufficient for violation of the noncontextuality inequality (3) for some quantum state.

Proof's scheme. Consider the operator

$$
\hat{\Gamma}=\hat{X}_{1} \hat{X}_{2}+\hat{X}_{2} \hat{X}_{3}+\hat{X}_{3} \hat{X}_{4}-\hat{X}_{4} \hat{X}_{1} \text {. }
$$

Then we have

$$
\hat{\Gamma}^{2}=4+\left[\hat{X}_{1}, \hat{X}_{3}\right]\left[\hat{X}_{2}, \hat{X}_{4}\right]=4+\hat{M}_{13} \hat{M}_{34} .
$$

Then it is easy to show that $\left\|\hat{\Gamma}^{2}\right\|>4$, if and only if condition (14) holds. Finally, we note that

$$
\sup _{\|\psi\|=1} \mid\langle\psi|\hat{\Gamma}| \psi\rangle=\|\hat{\Gamma}\|=\sqrt{\left\|\hat{\Gamma}^{2}\right\|} .
$$

We remark that condition (14) is trivially satisfied for incompatible observables, if the state space and observables have the tensor product structure: $H=H_{13} \otimes H_{24}$ and

$$
\hat{X}_{i}=\hat{\mathbf{X}}_{i} \otimes I, \hat{X}_{j}=I \otimes \hat{\mathbf{X}}_{j},
$$

where

$$
\hat{\mathbf{X}}_{i}: H_{13} \rightarrow H_{13}, i=1,3, \hat{\mathbf{X}}_{j}: H_{24} \rightarrow H_{44}, j=2,4 .
$$

Here condition (14) is reduced to incompatibility condition:

$$
\left[\hat{\mathbf{X}}_{i},: \hat{\mathbf{X}}_{j}\right] \neq 0, i=1,3 ; j=2,4 \text {. }
$$

In particular, for compound systems, contextuality ("nonlocality") is exactly incompatibility. The same is valid for any tensor decomposition of the state space of a single quantum system with observables of the type (18). In the tensor product case, contextuality without incompatibility leads to the notion with the empty content.

But, it may happen that $X_{i}$-observables, $i=1,3$, and $X_{j}$-observables, $j=2,4$, are not connected via the tensor product structure. In this case, the interpretation of constraint (14) is nontrivial. What is its physical meaning? I have no idea.

Of course, the main problem is that it is not clear at all how to measure the observables of the commutator-type.

\section{Conclusion}

In quantum physics, there is no contextuality without incompatibility. This is well known, but not so highly emphasized feature of quantum observables.

For fourth quantum observables, these two notions coincide under validity of constraint (14). If it is violated, then, for such observables, there is still a hope that quantum contextuality without incompatibility has some nontrivial physical meaning. (What?) The problem of nontrivial physical meaning of "pure contextuality", i.e., one distilled from incompatibility, for $n>4$ observables (as well as $n=3$, see Appendix 2) is open.

Finding the right physical interpretation for contextuality beyond incompatibility is important for demystification of quantum physics (cf. with discussion of Svozil [23] on "quantum focus pocus").

Funding Open access funding provided by Linnaeus University. 
Open Access This article is licensed under a Creative Commons Attribution 4.0 International License, which permits use, sharing, adaptation, distribution and reproduction in any medium or format, as long as you give appropriate credit to the original author(s) and the source, provide a link to the Creative Commons licence, and indicate if changes were made. The images or other third party material in this article are included in the article's Creative Commons licence, unless indicated otherwise in a credit line to the material. If material is not included in the article's Creative Commons licence and your intended use is not permitted by statutory regulation or exceeds the permitted use, you will need to obtain permission directly from the copyright holder. To view a copy of this licence, visit http://creativecommonshorg/licenses/by/4.0/.

\section{Appendix 1: Structuring Bohr's Contextuality and Complementarity Into a Single Principle}

As was emphasized in [27], the complementarity principle is closely coupled with the notion of contextuality that is understood in Bohr's sense. Bohr did not use the notion "experimental context". He operated with the notion of experimental condition [24]:

"Strictly speaking, the mathematical formalism of quantum mechanics and electrodynamics merely offers rules of calculation for the deduction of expectations pertaining to observations obtained under well-deffined experimental conditions specified by classical physical concepts."

Unfortunately, Bohr did not formulate his views on quantum foundations in the form of principles, similar to Einstein's principles of relativity. These views were presented in the form of the foundational statements connected with long texts on the general structure of quantum theory and its methodology, especially methodology of quantum measurements. And these statements were often modified year to year. Nevertheless, careful reading of Bohr's works leads to clear picture of quantum foundations. We remark that in this picture there is nothing mystical or too much surprising. This is logically well structured reasoning on specialty of quantum measurements (and, for Bohr, the quantum theory is a measurement theory). In my previous papers, I called this bunch of Bohr's views the complementarity principle. This can lead to misunderstanding. Nowadays, the complementarity principle is typically reduced to the wave-particle duality - the existence of incompatible observables (experimental contexts). The latter is just the concluding accord of long Bohr's play, the play on contextuality of quantum measurements. Since this paper is devoted to contextuality, this is the good place to restructure my formulation [9, 11, 27] of the Bohr's complementarity principle [24] - to highlight its contextual counterpart.

We start with pointing to the physical basis of quantum contextuality and complementarity. Bohr stressed $[37,38]$ that the essence of the quantum theory "may be expressed in the so-called quantum postulate, which attributes to any atomic process an essential discontinuity, or rather individuality, completely foreign to the classical theories and symbolised by Planck's quantum of action." This postulate is about nature as it is. And the postulate is the root of the fundamental principles of the quantum theory (the quantum measurement theory).

We continue with the famous citation of Bohr that presents the essence of his views on contextuality and complementarity of quantum measurements, see Bohr ([24], v. 2, p. 40-41):

"This crucial point ... implies the impossibility of any sharp separation between the behaviour of atomic objects and the interaction with the measuring instruments which serve to define the conditions under which the phenomena appear. In fact, the individuality of the typical quantum effects finds its proper expression in the circumstance that any attempt of 
subdividing the phenomena will demand a change in the experimental arrangement introducing new possibilities of interaction between objects and measuring instruments which in principle cannot be controlled. Consequently, evidence obtained under different experimental conditions cannot be comprehended within a single picture, but must be regarded as complementary in the sense that only the totality of the phenomena exhausts the possible information about the objects."

By the quantum postulate there exists indivisible quantum of action given by the Planck constant $h$. Its presence prevents approaching the internal features of a quantum system. Therefore it is meaningless (from the viewpoint of physics) to build scientific theories about such features. This reasoning (rooted in the quantum postulate) implies:

Principle of Contextuality The output of any quantum observable is indivisibly composed of the contributions of the system and the measurement apparatus.

There is no reason to expect that all experimental contexts can be combined and all observables can be measured jointly. Hence, incompatible observables (complementary experimental contexts) may exist. Moreover, they should exist, otherwise the contextuality principle would have the empty content. Really, if all experimental contexts can be combined into single context $\mathcal{C}$ and all observables can be jointly measured in this context, then the outputs of such joint measurements can be assigned directly to a system. To be more careful, we have to say: "assigned to a system and context $\mathcal{C}$ ". But, the latter can be omitted, since this is the same context for all observables. This reasoning implies:

Principle of Complementarity There exist incompatible observables (complementary experimental contexts).

Since both principles, contextuality and complementarity, are so closely interrelated, it is natural to unify them into the single principle, Contextuality-Complementarity principle.

Bohr's viewpoint on contextuality and its coupling with complementarity was explored in a series of author's papers, see, e.g., monograph [26].

This is the right place to stress once again the difference between Bohr-contextuality and the notion of contextuality that is widely used in considerations related to the Bell-type inequalities, Bell-contextuality [1,2]. ${ }^{3}$ The former has no relation to joint measurement. It is about context-dependence of outputs of a single observable. Of course, joint measurement of a compatible observable can also be considered as specification of experimental context. However, such viewpoint on contextualization only overshadow the original Bohr's view: contextuality as impossibility to separate (in measurement's output) the contributions of the system and measurement device.

We remark that coupling of the contextuality principle to the quantum postulate, the existence of the Planck constant, is important only for foundations of quantum physics. Outside of physics, one can start directly with the contextuality principle. It can be applied even to nonphysical systems, see, e.g., [17-20] on applications to decision making, cognitive and social sciences. However, the class of observables described by quantum mechanics is very special; they are represented by Hermitian operators acting in compelx Hilbert space, see Section 2 for foundational consequences.

In the line of the above reasoning (I hope that Bohr would agree with it), the existence of complementary experimental contexts and incompatible observables is very natural, there is nothing mystical in this. This is a consequence of (Bohr-)contextuality and the latter in turn

\footnotetext{
${ }^{3}$ We remark that Bell did not use the term "contextuality".
} 
is a consequence of the quantum postulate. Since we claim that quantum nonocality and Bell-contextuality are reduced to the existence of incompatible observables - the principle of complementarity, it seems that the only mystery of quantum physics is the quantum postulate (see [39] for details).

\section{Appendix 2: Suppes-Zanotti Inequality: Has it Any Relation to Quantum Physics?}

The case $n=3, X_{1}, X_{2}, X_{3}$, is special. Here the tight noncontextuality inequality was derived by Suppes and Zanotti [40]:

$$
\left\langle X_{1} X_{2}\right\rangle-\left\langle X_{2} X_{3}\right\rangle+\left\langle X_{1} X_{3}\right\rangle \leq 1
$$

Often this inequality is misleadingly coupled to the original Bell inequality. However, the Suppes-Zanotti inequality has nothing to do with quantum mechanics. Since it is assumed that all pairs of observables are compatible, the JPD for quantum observables always exists and this inequality is always satisfied. So, the criterion of the existence of JPD derived in [40] has no relation to quantum mechanics.

The original Bell inequality has the form:

$$
\left\langle X_{1} X_{2}\right\rangle-\left\langle X_{3} X_{4}\right\rangle+\left\langle X_{1} X_{4}\right\rangle \leq 1 .
$$

Here observable $X_{1}$ should be compatible with observables $X_{2}, X_{4}$ and $X_{3}$ with $X_{4}$. This is the inequality based on three contexts for four observables. It is not a tight noncontextuality inequality, so it is not covered by Theorem 1 [4]. Surprisingly this inequality is more complicated than inequality (3), see [41] for some steps towards its analysis.

\section{References}

1. Bell, J.S. Speakable and Unspeakable in Quantum Mechanics, 2nd edn. Cambridge University Press, Cambridge (2004)

2. Bell, J.S.: On the problem of hidden variables in quantum theory. Rev. Mod. Phys. 38, 450 (1966)

3. Cabello, A., Severini, S., Winter, A.: (Non-)contextuality of physical theories as an axiom. arXiv:1010.2163 [quant-ph]

4. Araujo, M., Quintino, M.T., Budroni, C., Cunha, M.T., Cabello, A.: All noncontextuality inequalities for then-cycle scenario. Phys. Rev. A 88, 022118 (2013). arXiv:1206.3212

5. Svozil, K.: What makes quantum clicks special? arXiv:1707.08915 [quant-ph]

6. Svozil, K.: "Haunted" quantum contextuality. arXiv:quant-ph/9907015

7. Svozil, K.: On counterfactuals and contextuality. In: AIP Conference Proceedings 750. Foundations of Probability and Physics-3, pp. 351-360. American Institute of Physics, Melville (2005). arXiv:quant-ph/0406014

8. Svozil, K.: Proposed direct test of a certain type of noncontextuality in quantum mechanics. Phys. Rev. A 80, 04010 (2009)

9. Khrennikov, A.: Get rid of nonlocality from quantum physics. Entropy 21(8), 806 (2019)

10. Khrennikov, A.: Quantum versus classical entanglement: eliminating the issue of quantum nonlocality. Found. Phys. https://doi.org/10.1007/s10701-020-00319-7. arXiv:1909.00267v1 [quant-ph] (2020)

11. Khrennikov, A.: Two faced Janus of quantum nonlocality. Entropy 22(3), 303 (2020). arXiv:2001.02977 [quant-ph]

12. Kupczynski, M.: Can Einstein with Bohr debate on quantum mechanics be closed. Phil. Trans. Royal Soc. A 375, 2016039 (2017)

13. Griffiths, R.B.: Quantum nonlocality: Myth and reality. arXiv:1901.07050 (2019)

14. Boughn, S.: Making sense of Bell's theorem and quantum nonlocality. Found. Phys. 47, 640-657 (2017) 
15. Hess, K.: Categories of nonlocality in EPR theories and the validity of Einstein's separation principle as well as Bell's theorem. J. Modern Phys. 10(10), 1209-1221 (2019)

16. Cetto, A.M., Valdes-Hernandez, A., de la Pena, L.: On the spin projection operator and the probabilistic meaning of the bipartite correlation function. Found. Phys. 50, 27-39 (2020)

17. Conte, E., Khrennikov, A., Todarello, O., Federici, A.: A preliminary experimental verification on the possibility of Bell inequality violation in mental states. Neuroquantology 6(3), 214-221 (2008)

18. Asano, M., Khrennikov, A., Ohya, M., Tanaka, Y., Yamato, I.: Quantum Adaptivity in Biology: from Genetics to Cognition. Springer, Berlin (2015)

19. Cervantes, V.H., Dzhafarov, E.N.: Snow Queen is evil and beautiful: Experimental evidence for probabilistic contextuality in human choices. Decision 5, 193-204 (2018)

20. Basieva, I., Cervantes, V.H., Dzhafarov, E.N., Khrennikov, A.: True contextuality beats direct influences in human decision making. arXiv:1807.05684 [q-bio.NC]

21. Moore, E.F.: Gedanken-experiments on sequential machines. Automata studies. In: Shannon, C.E., McCarthy, J. (eds.) Annals of Mathematics Studies, pp. 129-153. Princeton Univ. Press (1956)

22. Wright, R.: Generalized urn models. Found. Phys. 20, 881-903 (1990). https://doi.org/10.1007/BF0188 9696

23. Svozil, K.: Logical equivalence between generalized urn models and finite automata. Int. J. Theor. Phys. 44, 745-754 (2005). arXiv:quant-ph/0209136

24. Bohr, N.: The Philosophical Writings of Niels Bohr. Ox Bow Press, Woodbridge (1987)

25. Plotnitsky, A.: Niels Bohr and Complementarity: An Introduction. Springer, Berlin (2012)

26. Khrennikov, A.: Contextual Approach to Quantum Formalism. Springer, Berlin (2009)

27. Khrennikov, A.: Bohr against Bell: complementarity versus nonlocality. Open Phys. 15, 734-738 (2017)

28. von Neuman, J.: Mathematical Foundations of Quantum Mechanics. Princeton Univ Press, Princenton (1955)

29. Fine, A.: Joint distributions, quantum correlations, and commuting observables. J. Math. Phys. 23, 1306 (1982)

30. Kupczynski, M.: Bertrand's paradox and Bell's inequalities. Phys. Lett. A 121, 205-207 (1987)

31. De Muynck, W.: Interpretations of quantum mechanics and interpretations of violation Bell's inequality. In: Proc. Conference "Foundations of Probability and Physics", Växjö, 2000; WSP, Singapore (2001)

32. Nanasiova, O.: Map for simultanous measuremants for a quantum logic. Int. J. Theor Phys. 42, 18891903 (2003)

33. Khrennikov, A.: Bell-Boole inequality: Nonlocality or probabilistic incompatibility of random variables? Entropy 10, 19-32 (2008)

34. Nanasiova, O., Kalina, M.: Calculus for non-compatible observables, construction through conditional states. Int. J. Theor. Phys. 54, 506-518 (2015)

35. Khrennikov, A., Alodjants, A.: Classical (local and contextual) probability model for Bohm-Bell type experiments: No-Signaling as independence of random variables. Entropy 21, 157-177 (2019)

36. Lopez-Saldívar, J.A., Castanos, O., Nahmad-Achar, E., Lopez-Pena, R., Man'ko, M.A., Man'ko, V.I.: Geometry and entanglement of two-qubit states in the quantum probabilistic representation

37. Bohr, N.: The quantum postulate and the recent development of atomic theory. Supplement to Nature, 580-590 (1928)

38. Bohr, N.: The quantum of action and the description of nature. In: Kalckar, J. (ed.) Foundations of Quantum Physics I (1926-1932). Niels Bohr collected works, vol. 6, pp. 201-217. Elsevier B.V. (1985)

39. Khrennikov, A.: Quantum postulate vs. quantum nonlocality: Is Devil in h? arXiv:2003.05718 [quant-ph] 40. Suppes, P., Zanotti, M.: Synthese 48, 191 (1981)

41. Loubenets, E.R., Khrennikov, A.Y.: Quantum analog of the original Bell inequality for two-qudit states with perfect correlations/anticorrelations. J. Phys. A Math. Theor. 52, 435304 (2019)

Publisher's Note Springer Nature remains neutral with regard to jurisdictional claims in published maps and institutional affiliations. 